\title{
New Insights into Deformation of Metallic Glasses by Combining Mesoscale Simulation and Fluctuation Electron Microscopy
}

\author{
Pengyang Zhao ${ }^{1}$, Soohyun $\operatorname{Im}^{1}$, Jinwoo Hwang ${ }^{1}$, and Yunzhi Wang ${ }^{1}$ \\ 1. Department of Materials Science and Engineering, The Ohio State University, Columbus, OH 43212
}

We present a new mesoscale deformation model of metallic glasses (MGs) that incorporates nanoscale atomic ordering information from fluctuation electron microscopy (FEM). Understanding the deformation mechanism of MGs, including shear banding at room temperature, is crucial to overcome their limited ductility and early failure, which have been a major obstacle to widespread applications of MGs. The current understanding attributes shear band initiation to collective interaction among shear transformation zones (STZs) where shear deformation occurs among a group of atoms in a nanoscale volume [1]. We previously showed that mesoscale simulation involving diverse STZ types can provide useful insights into shear banding and overall deformation characteristics of MGs beyond the time and length scale of atomistic simulations [2]. Here we demonstrate that more realistic deformation simulations can be achieved by incorporating the experimentally measured medium range ordering (MRO) information using FEM directly into the heterogeneously randomized STZ environment in our model. Our approach is based on the assumption that each MRO type, which resides at the same length scale of STZ, will have a different set of parameters that characterize the STZ events, which has also been indicated by others [see, e.g., 3].

FEM is based on electron nanodiffraction (Figure 1a and b), and it can provide the details of MRO, including size, type, volume fraction, distribution, and networking [4]. For example, FEM has discovered new "crystal-like” $\mathrm{MRO}$ in $\mathrm{Zr}_{50} \mathrm{Cu}_{45} \mathrm{Al}_{5} \mathrm{MG}$, and its relaxation into MRO with plane-like structure upon annealing (Figure 1c) [5]. Incorporating such new MRO structures into mesoscale simulation can therefore provide useful insights on how the distribution and volume fraction of such MRO affect the changes in mechanical properties, such as the loss of ductility after annealing.

Based on the experimental FEM result [5], we have incorporated crystal-like STZs (cSTZs) that exhibit pseudo-FCC symmetry into the matrix glass that consists of random ("glassy") STZs (Figure 2a). Realistic estimation of the number of STZ slip modes and activation energy is important. Here we assume that the cSTZ has 6 slip modes, which are less than the 20 modes assumed in glassy STZs. The activation energy of cSTZ is inherently correlated with the symmetry of MROs and/or the local stiffness [3]. The volume fraction of cSTZ is estimated from the experimental FEM data [6]. All STZs are $1.7 \mathrm{~nm}$ in size, and spatially distributed with random orientations in a $0.44 \times 0.87 \mu \mathrm{m}^{2}$ box. Kinetic Monte Carlo (KMC) algorithm is used to simulate the STZ dynamics. Model parameters are calibrated using experimental stress-strain curves [e.g. 7]. Failure is not considered, as the main focus of the current investigation is to study continued shear bands' growth and thickening after their nucleation. Effects of the volume fraction of cSTZs, softening behavior of cSTZs, and MRO symmetry and its correlation with activation barriers are investigated parametrically for uniaxial tension in two-dimensions (2D), which exhibit a strong correlation between MRO properties and mechanical behaviors. For example, the simulation shows that increasing the volume fraction of cSTZ can noticeably lower the yield stress (Figure 2b) and increase the diffuseness and homogeneity of the shear bands (Figure 2c). The latter implies that incorporation of crystal-like MRO may improve ductility. Using extreme value statistics analysis [2], the exact change in the ductility can be quantified, which can then be compared directly to 
the experimentally measured ductility. The combination of FEM and the heterogeneously randomized STZ model is therefore a promising new method that may provide a new understanding on shear banding and deformation of MGs. New results obtained from $\mathrm{Zr}-\mathrm{Cu}-\mathrm{Co}-\mathrm{Al}$ MGs will also be discussed.

\section{References}

[1] M.L. Falk and J.S. Langer, Annual Review of Condensed Matter Physics, 2, 353 (2011).

[2] P. Zhao, J. Li, and Y. Wang, Acta Materialia, 73, 149 (2014).

[3] J. Ding, S. Patinet, M.L. Falk, Y. Cheng, and E. Ma, PNAS, 111, 14052 (2014).

[4] M.M.J. Treacy et al., Rep. Prog. Phys., 68, 2899 (2005).

[5] J. Hwang et al., Phys. Rev. Lett., 108, 195505 (2012).

[6] J. Hwang and P.M. Voyles, Microscopy and Microanalysis, 17, 67 (2011).

[7] G. Kumar et al., Scripta materialia, 57, 173 (2007).
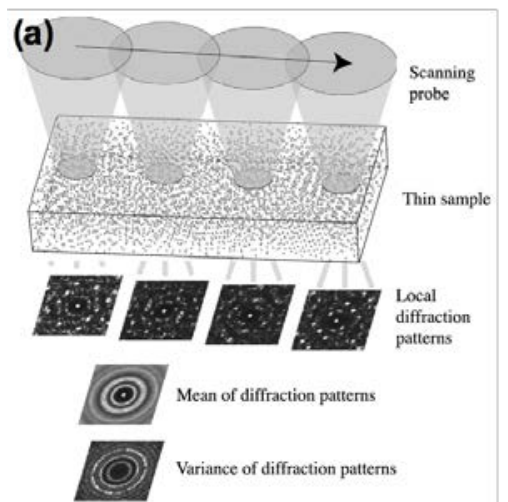
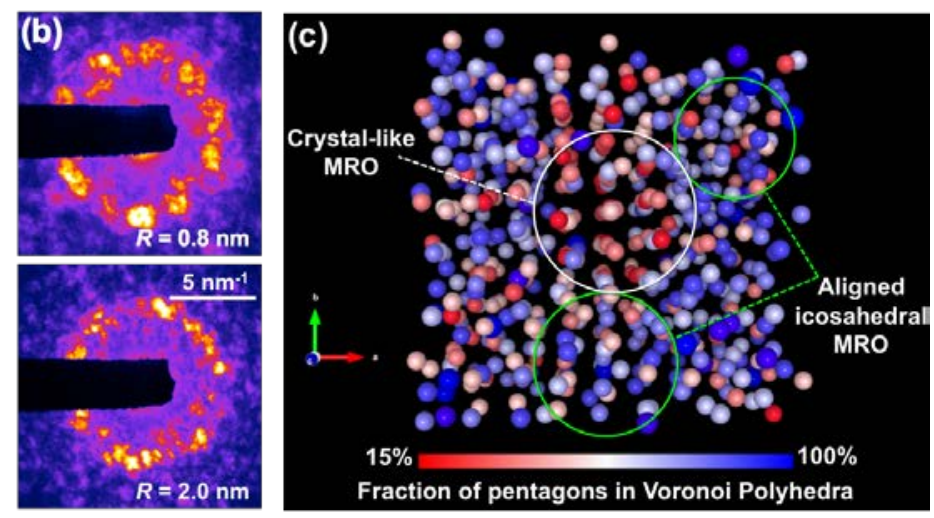

Figure 1. (a) A typical scattering geometry for FEM in STEM [4], and (b) FEM nanodiffraction patterns from an MG [6]. (c) MRO model generated based on FEM data from $\mathrm{Zr}_{50} \mathrm{Cu}_{45} \mathrm{Al}_{5} \mathrm{MG}$ [5].

(a)
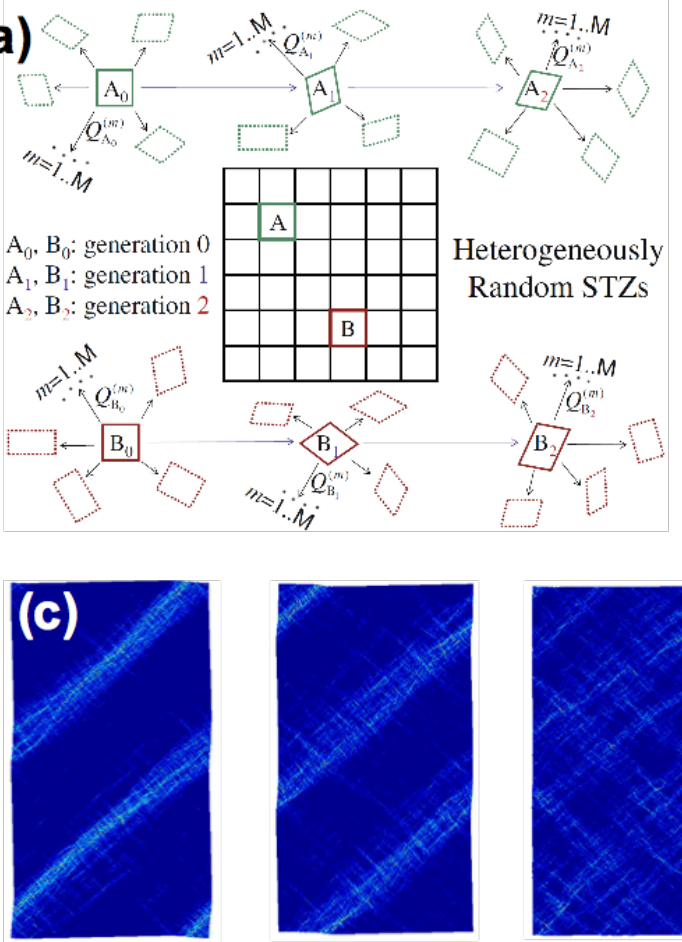

$\mathrm{C}=\mathbf{0}$

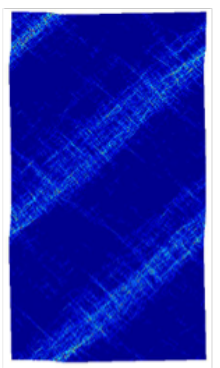

$\mathrm{C}=0.2$

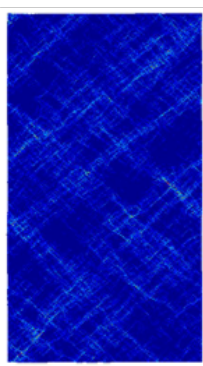

$C=0.5$ (b)

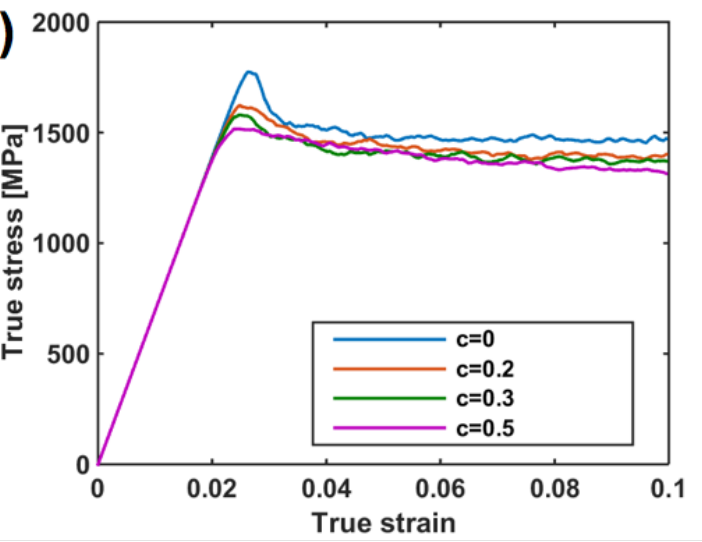

Figure 2. (a) Illustration of the heterogeneously randomized STZ model. $Q(m)$ is the activation free energy for a voxel to transform in the $m$-th of the total M modes [2]. (b) Tensile stress-strain curve and (c) shear banding patterns from the mesoscale simulation incorporating the crystallike MRO measured using FEM. $c$ is the volume fraction of the MRO. 